\title{
Sending hope to Mars
}

\author{
The Emirates will shortly join the ranks of space-faring nations by sending an orbiter to Mars, aiming to make novel \\ observations and inspire the country, write the mission leadership team.
}

T he coming month is set to mark the launch of three spacecraft bound for our near-neighbour, Mars. One of them will see the United Arab Emirates (the Emirates) as a new entrant into planetary exploration, with the Emirates Mars Mission (EMM) launching the Mars Hope Probe (Fig. 1), which will begin orbiting Mars in February 2021. The primary motivations for the mission are to further develop the Emirates' capabilities in designing and developing complex systems, and to expand scientific capacity to enable deeper innovation across more scientific fields, thereby creating new opportunities for engineers, scientists and researchers. This mission is one of several national programmes launched by the Emirates government to transition into an economy based on science and technology.

Development started on EMM in early 2014, with the primary objective of developing a spacecraft with novel scientific goals that filled a gap in our understanding of Mars, rather than a mission that solely demonstrated technological capabilities. The Emirates has successfully developed and launched Earth-observation satellites, but EMM proved from the start to be much more complex than any prior mission. A joint-development approach was taken, utilizing the internal capabilities at the Mohammed bin Rashid Space Centre (MBRSC) and bridging the expertise gap through an international knowledge collaboration with the Laboratory for Space and Planetary Physics at the University of Colorado, Boulder, the School of Earth and Space Exploration at Arizona State University, and the Space Science Lab at the University of California, Berkeley.

The Hope Probe carries three instruments developed jointly with the scientific partners: the Emirates eXploration Imager (EXI), Emirates Mars Infrared Spectrometer (EMIRS) and Emirates Mars Ultraviolet Spectrometer (EMUS). The EXI instrument is a multi-wavelength imager capable of capturing $12 \mathrm{MP}$ visible images of Mars, along with observations in the spectral ranges $245-275 \mathrm{~nm}$ and $305-335$ $\mathrm{nm}$. The EMIRS instrument characterizes the lower atmosphere in conjunction with EXI, observing in the $6-40 \mu \mathrm{m}$ spectral range, while EMUS observes the upper atmosphere with a 100-170 $\mathrm{nm}$ spectral window. The integrated observations

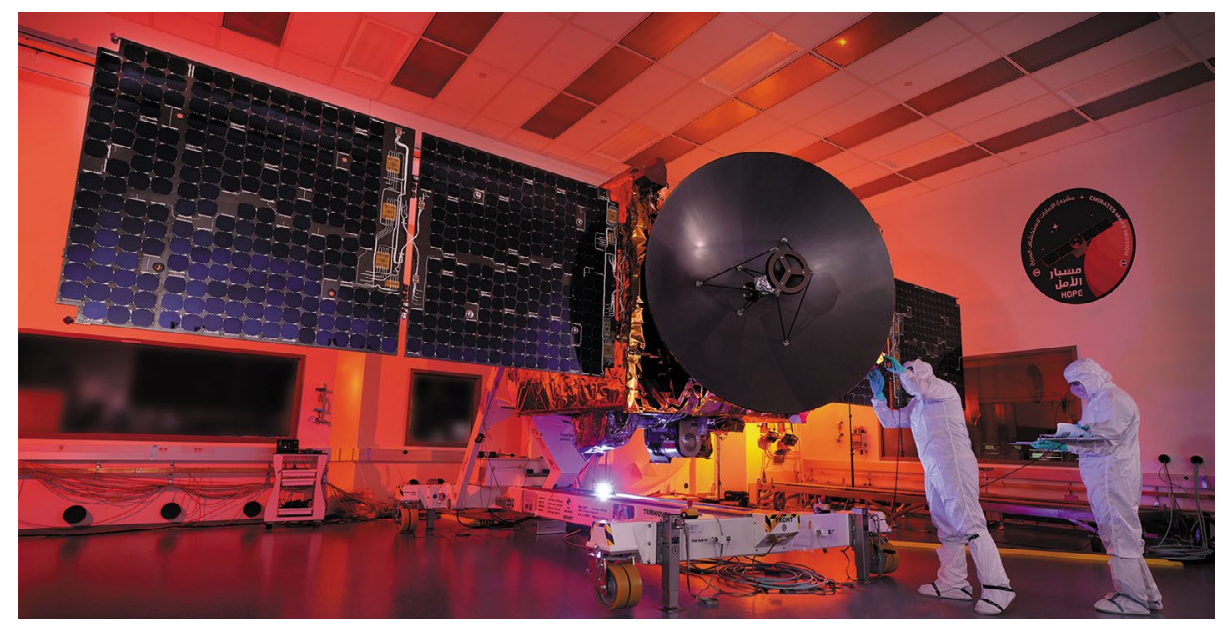

Fig. 1| The Mars Hope Probe in the final stages of preparation. Credit: MBRSC.

from these instruments will satisfy three scientific objectives. First, the Hope Probe will characterize the weather system in the lower atmosphere of Mars, providing a comprehensive understanding of diurnal changes across the planet and across all seasons. The unique orbit of the spacecraft (20,000 km periapsis and 43,000 km apoapsis, with an orbital period of 55 hours and orbital inclination of $25^{\circ}$ ) will allow the sampling of nearly all regions of Mars at every time of day, every $\sim 10$ days. No other Mars spacecraft has had such an orbit, enabling unprecedented studies of the physical processes that drive the distribution and interaction of ice clouds, water vapour and dust in the lower atmosphere. Second, EMM will measure the distribution of hydrogen and oxygen in the highest portions of the atmosphere (the collisionless exosphere). These species are known to escape to space from the planet ${ }^{1}$, a process that we believe may have been responsible for Mars's transition, over billions of years, from having a thick atmosphere capable of sustaining liquid water on the surface to the cold, thin and arid atmosphere we see today. Finally, the Hope Probe will correlate the processes within the lower atmosphere with rates of atmospheric escape from the exosphere, giving us a comprehensive understanding of the connections between weather change and atmospheric loss. These connections have been recently suspected to act more rapidly and be more complex than previously thought ${ }^{2,3}$.
The Hope Probe is scheduled to launch on a Mitsubishi Heavy Industries H-IIA launch vehicle from Tanegashima Space Centre, Japan. The launch window opens on 14 July 2020 (UTC), and launch will be followed by a seven month cruise to Mars. Over roughly two months, instrument checks from a high orbit will provide early observations of the Mars disk and upper atmosphere. In April 2021, the spacecraft will transition into its final science orbit. The primary science mission will last for one Martian year, with a possible extension of another. Data from the mission will be available to the entire science community through the Emirates Science Data Centre website without an embargo period, with the first set of data products available between August and September 2021. It is our hope that the mission will inspire a country, and help us to better understand the Martian atmosphere for years to come.

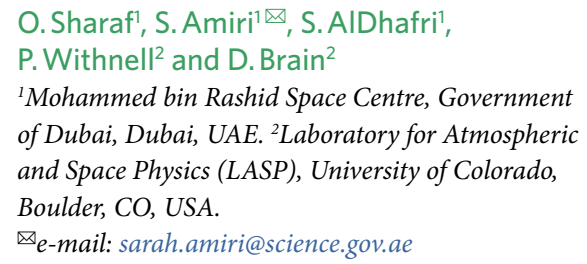
of Dubai, Dubai, UAE. ${ }^{2}$ Laboratory for Atmospheric and Space Physics (LASP), University of Colorado, Boulder, CO, USA.

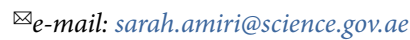

Published online: 13 July 2020 https://doi.org/10.1038/s41550-020-1151-y

\section{References}

1. Jakosky, B. M. et al. Icarus 315, 146-157 (2018).

2. Chaffin, M. S. et al. Geophys. Res. Lett. 41, 314-320 (2014).

3. Clarke, J. T. et al. Geophys. Res. Lett. 41, 8013-8020 (2014). 